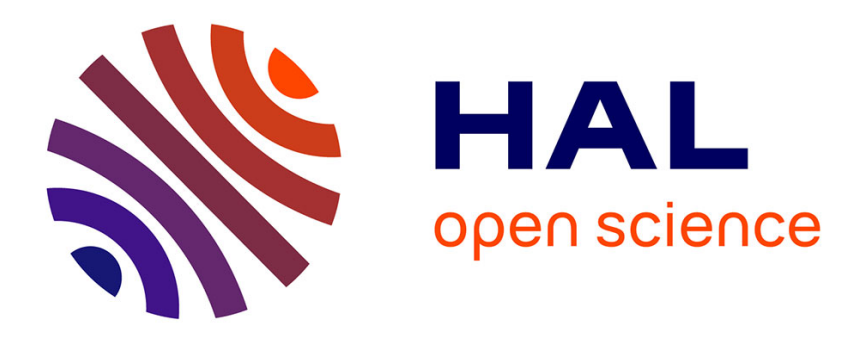

\title{
The validity of hyperscaling in three dimensions for scalar spin systems
}

\author{
M. E. Fisher, Jing-Huei Chen
}

\section{To cite this version:}

M. E. Fisher, Jing-Huei Chen. The validity of hyperscaling in three dimensions for scalar spin systems. Journal de Physique, 1985, 46 (10), pp.1645-1654. 10.1051/jphys:0198500460100164500 . jpa00210112

\section{HAL Id: jpa-00210112 https://hal.science/jpa-00210112}

Submitted on 1 Jan 1985

HAL is a multi-disciplinary open access archive for the deposit and dissemination of scientific research documents, whether they are published or not. The documents may come from teaching and research institutions in France or abroad, or from public or private research centers.
L'archive ouverte pluridisciplinaire HAL, est destinée au dépôt et à la diffusion de documents scientifiques de niveau recherche, publiés ou non, émanant des établissements d'enseignement et de recherche français ou étrangers, des laboratoires publics ou privés. 
Classification

Physics Abstracts

$05.50-05.70 \mathrm{~J}-75.40 \mathrm{D}$

\title{
The validity of hyperscaling in three dimensions for scalar spin systems
}

\author{
M. E. Fisher (*) \\ Department of Theoretical Physics, University of Oxford, 1 Keble Road, Oxford OX1 3NP, U.K. \\ and Jing-Huei Chen \\ Baker Laboratory, Cornell University, Ithaca, New York 14853, U.S.A.
}

(Reçu le 28 mai 1985, accepté le 3 juin 1985)

\begin{abstract}
Résumé. - Nous calculons les exposants $\gamma, v$ et $\alpha$ à partir des développements en série de la longueur de corrélation et de la susceptibilité magnétique dans les modèles double gaussien et de Klauder sur réseaux cubiques centrés. Ces modèles interpolent en fonction d'un paramètre $y$ entre le modèle gaussien pur $(y=0)$ et le modèle d'Ising de spin $1 / 2(y=1)$. Une analyse à une variable suggère l'existence d'une valeur $y_{\mathrm{c}}$ (pour chaque modèle) pour laquelle la première correction non analytique à une pure loi de puissance s'annule. Ce résultat est en accord avec une analyse antérieure utilisant des approximants différentiels à deux variables. Les hamiltoniens des deux modèles ont des caractéristiques quasi universelles en ces points. Nous en déduisons une estimation pour les exposants critiques : $\gamma=1,2395 \pm 0,0004, v=0,632 \pm 0,001, \alpha=0,105 \pm 0,007$ : ceci implique $d v-(2-\alpha)=0,001 \pm 0,010$ et indique que l' « hyperscaling " est vérifié. Des déviations apparentes observées dans le modèle d'Ising pur peuvent être attribuées à des corrections non analytiques petites, mais non négligeables.
\end{abstract}

\begin{abstract}
The exponents $\gamma, v$ and $\alpha$ are estimated from susceptibility and correlation length series for the bcc double-Gaussian and Klauder models, which interpolate, as a function of $y$, between the pure Gaussian model (at $y=0$ ) and the pure spin 1/2 Ising model (at $y=1$ ). Single-variable analysis reveals a value, $y_{\mathrm{c}}$ (for each model), at which the leading, non-analytic corrections to pure power laws vanish, in accordance with expectations based on prior partial differential approximant analysis. The Hamiltonians of the two models display quasiuniversal features at these points. The corresponding exponent estimates are $\gamma=1.2395 \pm 0.0004, v=0.632 \pm 0.001$ and $\alpha=0.105 \pm 0.007$ : these imply $d v-(2-\alpha)=0.001 \pm 0.010$ and hence indicate the validity of hyperscaling. Apparent violations seen in the pure Ising model are attributable to small but significant nonanalytic corrections.
\end{abstract}

\section{Introduction and summary.}

The phenomenological scaling laws for critical exponents fall into two independent classes. On the one hand are the ordinary relations like

$$
\begin{aligned}
\alpha+2 \beta+\gamma & =2, \quad \Delta=\beta \delta=\beta+\gamma, \\
\gamma & =(2-\eta) v,
\end{aligned}
$$

connecting the thermodynamic exponents, $\alpha, \beta, \gamma, \delta$ and $\Delta$, the correlation length exponent, $v$, and the

(*) Permanent address : Baker Laboratory, Cornell University, Ithaca, New York 14853, U.S.A. critical point decay exponent, $\eta[1]$; on the other hand are the so-called hyperscaling relations [2]

$$
\begin{gathered}
d v=2-\alpha, \\
d(\delta-1) /(\delta+1)=2-\eta, \quad d v=2 \Delta-\gamma,
\end{gathered}
$$

which depend explicitly on the spatial dimensionality, $d$. The ordinary relations (1) are satisfied by all soluble models, including mean field theory and the spherical model for general $d$; the hyperscaling relations are found to be valid for soluble two-dimensional models, in particular for the pure, spin $\frac{1}{2}$ Ising model but they certainly fail in more than four dimensions where, 
e.g. for the spherical model, one finds the discrepancy

$$
\omega^{*} v \equiv d v-(2-\alpha)=\frac{1}{2}(d-4), \quad(d \geqslant 4) .
$$

The $(d=3)$-dimensional pure Ising model has long been studied on the basis of series expansions [3]. The ordinary scaling laws are well confirmed by estimates of the exponents derived by extrapolating the series but the most careful work prior to 1980 suggested that hyperscaling failed. Thus, using 11 or 12 terms for various lattices the initial estimates were [3]

$$
\begin{gathered}
\alpha=0.125 \pm 10, \quad \gamma=1.250 \pm 3, \\
v=0.638(+3,-2)
\end{gathered}
$$

where here, as everywhere below, the quoted uncertainties refer always to the last decimal place displayed. These estimates yield the small but apparently significant discrepancy

$$
\omega^{*} v=0.039(+19,-16) .
$$

In later work Baker [4] tested the hyperscaling relation (3) for $\Delta$ by studying the fourth field derivative, $\left(\partial^{2} \chi / \partial H^{2}\right)$, which diverges as $\left(T-T_{\mathrm{c}}\right)^{-\gamma-2 \Delta}$; he concluded that there was a discrepancy $d v-(2 \Delta-\gamma)$ $=0.028 \pm 3$ which, through ordinary scaling, is consistent with (6). The positivity of these deviations is also in accord with the validity of the ordinary relations (1) and the Gunton-Buckingham inequality, which replaces $=$ by $\geqslant$ in the first member of (3).

The apparent failure of hyperscaling for $d=3$ became a sharper problem with the advent of renormalization group theory [e.g. 5,6$]$ since, at the purely formal level, the theory necessarily entails hyperscaling. Furthermore, hyperscaling is verified explicitly to all orders of the $\varepsilon=4-d$ expansion for exponents and hence is expected to be valid for $d=3$ [7]. Note that the failure of hyperscaling for $d>4$ can be understood within renormalization group theory in terms of a dangerous irrelevant variable $[6,8]$ which enters into the scaling functions for the free energy, etc. ; however, there is no independent evidence for the presence of any dangerous variables in $d=3$ dimensions. In addition, direct application of field-theoretic renormalization group techniques to Ising-like $(n=1)$ scalar spin systems in $d=3$ yielded the estimates $[9,10]$

$$
\begin{gathered}
\alpha=0.110 \pm 5, \quad \gamma=1.241 \pm 2, \\
v=0.630 \pm 2
\end{gathered}
$$

(which necessarily verify hyperscaling). Since most theorists felt that the pure spin $\frac{1}{2}$ Ising model should be in the same universality class as the continuous, scalar spin models the difference in the estimates for $\gamma$ [compare with (5)] was disturbing and stimulated further studies. For example, Zinn-Justin [11] reanalysed the existing high temperature series for the pure Ising model concluding $\alpha=0.110 \pm 4$ and $\gamma=1.245 \pm 3$ in more satisfactory agreement with (7). However, since his estimate for $v$ was unchanged from (5), a discrepancy $\omega^{*} v=0.024 \pm 10$ remained. Nickel and Sharpe [12] and Rehr [13] examined the renormalized coupling constant (defined in terms of $\partial^{2} \chi / \partial H^{2}$ ) again casting doubt on the reliability of earlier extrapolations ; consistency with hyperscaling was suggested by Rehr but with no great conviction.

The situation changed dramatically in 1980 when Nickel [14] derived high temperature series for the bcc lattice to order 21 in the spin-spin coupling, for models with a variety of single-spin weight functions. In particular, Nickel showed, using the susceptibility series for the spin- $S$ Ising models, that the shorter series previously available were definitely not long enough to reveal the true asymptotic trends or the unambiguous presence of the leading nonanalytic correction to scaling. This correction is characterized by an exponent $\theta$ and enters the asymptotic behaviour for the susceptibility as

$$
\chi(T) \approx C t^{-\gamma}\left[1+c_{\theta} t^{\theta}+c_{1} t+\cdots\right],
$$

where

$$
t=\left(T-T_{\mathrm{c}}\right) / T_{\mathrm{c}} .
$$

Nickel's initial analysis suggested $\theta \simeq 0.5$ (in accord with renormalization group estimates) and $\gamma \simeq 1.237 \pm 5$ in better consonance with (7). Zinn-Justin [15] made a very careful analysis of the spin-S series for $\chi$ and for the mean square correlation length, $\xi^{2}$. By assuming that the exponent $\theta$ was independent of $S$ he was able to convincingly justify the estimates $\gamma=1.2385 \pm 25$ and $v=0.6305 \pm 15$, both in remarkably good accord with the field-theoretic results (7). A later analysis of the same series by Ferer and Velgakis [16] attempted to avoid assumptions about $\theta$ and led to the somewhat higher estimates $\gamma=1.242(+3,-5)$ and $v=0.634(+3$, -4). These authors claimed that the nonanalytic corrections for $S=\frac{1}{2}$ are " absent or at least unobservably small ». We will take issue with that conclusion and demonstrate that, even in the pure spin $-\frac{1}{2}$ Ising model, there are nonanalytic corrections which are significant in that they yield relatively high effective values of $\gamma$ and $v$ such as found by Ferer and Velgakis.

More importantly, neither Zinn-Justin nor Ferer and Velgakis estimates $\alpha$ or $\Delta$ so that no check of hyperscaling per se has yet been presented on the basis of the long series calculated by Nickel. The work to be reported here closes this gap. In fact we claim more : specifically, we conclude that if proper allowance is made for the possible presence of nonanalytic corrections to the leading power laws then the validity of hyperscaling or, more concretely, the estimate

$$
\omega^{*} v \equiv d v-(2-\alpha)=0 \pm 0.01, \quad(d=3),
$$

is indicated independently of any explicit assumptions about the detailed form of the corrections or the value of $\theta$. 
The crux of our approach is the recognition [14, 15 , 17] that in analysing one single series expansion of any practically attainable length (say, 30 terms or fewer) it is normally impossible to reliably gauge the character, magnitude and influence of the nonanalytic corrections at the requisite level of precision. It follows that one should analyse more than one series for a given model but also, and more importantly, one should study a family of models (specified by some auxiliary parameter) which are good candidates for belonging to the same universality class. The spin-S Ising models provide one such family but it is not really an optimal choice : in the first place the spin parameter is essentially discrete whereas continuity is desirable, if as will be our aim, one hopes to locate a parameter value at which the leading nonanalytic corrections vanish. One can, in fact, formally regard the spin as a continuous parameter since $S$ enters the series coefficients in a simple polynomial fashion; however, the corresponding analytically-continued single-spin weighting factors (for continuous spin variables) necessarily vary rapidly with $S$ and, in between half-integral values, do not even remain positive [18]. Secondly, the spin-S Ising family contains no simple limiting models which might provide some sort of an anchor in analysing the possible universality classes. For these reasons we have preferred to study the double-Gaussian (DG) and Klauder (Kl) models [17] which are specified by (i) a nearest-neighbour Hamiltonian with coupling terms $-J s_{i} s_{j}$ and temperature parameter

$$
x \equiv K=J / k_{\mathrm{B}} T,
$$

and (ii) single-spin weighting factors $[17,19]$

$$
\begin{gathered}
W_{\mathrm{DG}}(s ; y) \propto b\left[\mathrm{e}^{-b^{2}(s-\sqrt{y})^{2}}+\mathrm{e}^{\left.-b^{2}\left(s+\sqrt[V y)^{2}\right]{ }\right],}\right. \\
W_{\mathrm{K} 1}(s ; y) \propto b|s|^{y /(1-y)} \mathrm{e}^{-b^{2}\left(s^{2}-1\right)},
\end{gathered}
$$

in which $y$ is the auxiliary model parameter which also enters via

$$
b^{2}(y)=1 / 2(1-y) .
$$

At $y=0$ both models reduce to the simple Gaussian model which is exactly soluble having $\gamma=1, v=\frac{1}{2}$ and $\alpha=\frac{1}{2}(4-d)$ (although the model is undefined for $T<T_{\mathrm{c}}$ ). When $y \rightarrow 1$ both models approach the pure spin $-\frac{1}{2}$ Ising model. Furthermore, the doubleGaussian model can be transformed exactly into a spin $-\frac{1}{2}$ Ising model with further-neighbour but exponentially decaying interactions [20] and this provides an analytical and physically sensible continuation of the model to $y>1$ so that $y=1$ is not a singular case. The assignment (14) normalizes the noninteracting single-spin moments, $\left\langle s^{2}\right\rangle_{0}$, to unity for both models. The cross-model identification

$$
y_{\mathbf{K}_{1}} \cong y_{\mathrm{DG}}^{2},
$$

ensures, in addition, the equality of the noninteracting fourth moments, with $\left\langle s^{4}\right\rangle_{0}^{\mathbf{K} 1}=3-2 y_{\mathrm{K} 1}$ [19]. Higher moments of the spin distributions do, of course, differ but for $y$ not too small, the two distributions (12) and (13) are quite similar when (15) is used. This may be judged from figure 1 in which $W(s ; \mathrm{y})$ is plotted for $y_{\mathrm{DG}}=0.90$ (bold curve) and $y_{\mathrm{K}_{1}}=0.81$ (thin solid curve). (The significance of this choice of values will appear below).

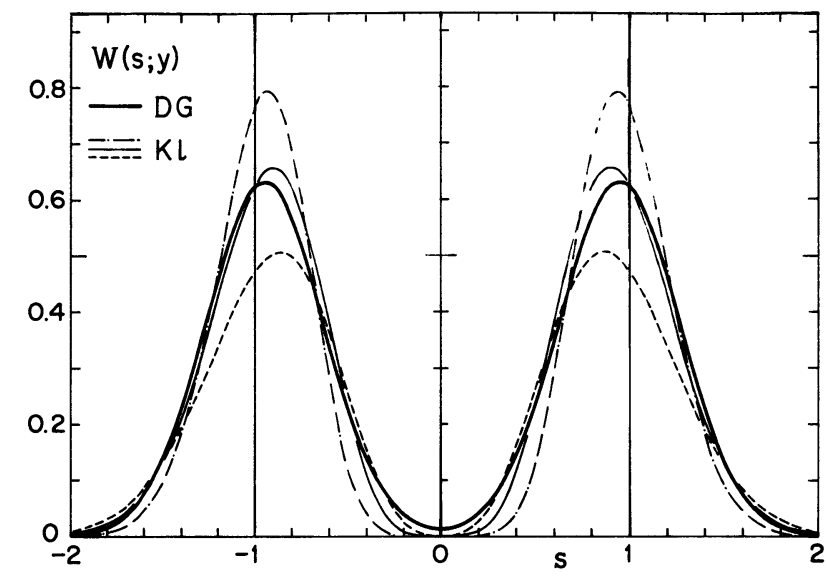

Fig. 1. - Single-spin weight factors for the double-Gaussian (DG) and Klauder (Kl) models normalized to have the same zeroth and second moments. The parameters used here are $y^{\mathrm{DG}}=0.90$ (bold solid curve) and $y^{\mathrm{K} 1}=0.81$ (thin solid curve) at which values the fourth moments of the two models are also the same, and $y^{\mathbf{K} 1}=0.87$ (dot-dash curve), 0.75 (dashed curve) : see further in section 2.

The double power series in $x$ and $y$ to order 21 for the susceptibility, $\chi(x, y)$, of the DG and $\mathrm{K} 1$ models on the bcc lattice have been studied previously [17] using two-variable partial differential approximants (PDAs) in order to achieve unbiassed estimates of the correction exponent $\theta$. The conclusions of this analysis, inasfar as they are relevant to our present purposes, are summarized in the next section. In particular, both models (for $y>0$ ) belong to only one Ising-like universality class but it is also clear that the leading corrections do not vanish for the pure Ising model. In section 3 we employ single-variable inhomogeneous differential approximants [21] at fixed $y$, to estimate the ferromagnetic critical locus, $x_{\mathrm{c}}(y)$, for each model using, first, the susceptibility series, second, the series for the second correlation moment, namely,

$$
\mu_{2}(x, y)=\sum_{i} R_{0 i}^{2}\left\langle s_{0} s_{i}\right\rangle,
$$

in which $R_{0 i}$ is the distance from the origin site, 0 , to site $i$, and, third, the series for the mean square correlation length,

$$
\xi^{2}(x, y)=\mu_{2}(x, y) / \chi(x, y) .
$$


For general values of $y$ the different series (and the imposition of various constraints) lead to estimates for $x_{\mathrm{c}}(y)$ differing by up to one or two parts in the fourth decimal place. However, for both models it is found that all the various methods agree to within only one part in $10^{5}$ of $x_{\mathrm{c}}$ when $y$ is in a narrow range lying, for each model, essentially at the multicritical point, $y_{c}$, as located in the earlier PDA analysis [17]. The agreement of different methods, all of which make no special allowance for nonanalytic corrections, at these special values of $y$ confirms the PDA and renormalization group expectations that the leading nonanalytic corrections should vanish at $y_{\mathrm{c}}$.

The exponents of $\gamma$ and $v$ are estimated in section 4 from the divergences $\chi \sim t^{-\gamma}, \mu_{2} \sim t^{-\gamma-2 v}$, and $\xi^{2} \sim t^{-2 v}$ for positive, i.e. ferromagnetic $x$; the specific heat exponent $\alpha$, on the other hand, is estimated from the behaviour at the symmetrically placed antiferromagnetic singularity, $x=-x_{\mathrm{c}}(y)$, at which one has

$$
f(x, y)=f_{\mathrm{c}}+A_{f} t^{1-\alpha}+B_{f} t+\cdots,
$$

for $f=\chi, \mu_{2}$ or $\xi^{2}$. This energy-like singular behaviour follows from various general considerations including the field-theoretic operator product expansion $[1,2,7$, 22]. The other point to notice is that such weak additive singularities can be reliably estimated (in the absence of further confluent nonanalyticities) by the use of inhomogeneous partial differential approximants since these allow naturally for an analytic background, $f_{\mathrm{c}}+B_{f} t+\cdots[21]$. It is found that the exponents $\gamma$, $v$ and $\alpha$ estimated in various ways also exhibit confluences close to the same special values of $y$. The corresponding exponents found from the DG and $\mathrm{Kl}$ models likewise agree with each other for these values of $y$ which, to well within the precision, also satisfy (15). The overall estimates obtained are then

$$
\begin{gathered}
\gamma=1.2395 \pm 4, \quad v=0.632 \pm 1, \\
\alpha=0.105 \pm 7,
\end{gathered}
$$

which, finally, lead to (10) and the confirmation of hyperscaling. (It should be mentioned that the uncertainties quoted here do not make full allowance for uncertainties in the location of $y_{\mathrm{c}}$ : see further in Sect. 4).

Finally, it is worth remarking that the estimates (19) apply for the whole range of the $\mathrm{DG}$ and $\mathrm{Kl}$ models (for $y>0$ ) including the pure Ising limit, and are in very satisfactory agreement with the field-theoretic $d=3$ renormalization group estimates (7) and, for $\gamma$ and $v$, in even better agreement with Zinn-Justin's estimates [15] for the spin-S Ising models as quoted after equation (8). Indeed, it seems likely that the lower estimates of $\gamma$, close to 1.239 [see also 17], and higher estimates of $v$, close to 0.631 , are nearer the true values than the $d=3$ field-theoretic estimates. Incidentally, the values (19) imply, via (1), $\eta=0.039 \pm 4$ whereas the field-theoretic calculations for $d=3$ yield $\eta=0.031 \pm 4[9,10]$. More recently, however, George and Rehr [23] have used a two-series approach to partial differential approximants for the doubleGaussian model and concluded $\gamma=1.2378 \pm 12$ and $v=0.6312 \pm 6$ and thence, $\eta=0.0375 \pm 10$ (where we have doubled the authors' one-standard-deviation quoted uncertainties to correspond better to the other precision estimates here). For completeness one should also note results based on the $\varepsilon$ expansion to fourth and fifth orders [24]. The latter were extrapolated to yield the surprisingly and, we suspect, unrealistically low estimates $\gamma=1.234 \pm 3$ and $v=0.628 \pm 1$, which, via (1), imply $\eta=0.035 \pm 8$. Of course, these $\varepsilon$ expansion results have hyperscaling built in. Lastly, recent Monte Carlo renormalization group calculations [25], which likewise entail hyperscaling, yield the estimates $v=0.629 \pm 4$ and $\eta=0.031 \pm 5$ and imply $\gamma=1.238 \pm 11$. However, these calculations also lead to an estimate of the correction exponent of $\theta \simeq 0.6_{3}$ which is out of line with all previous estimates, namely, $\theta=0.50 \pm 2(d=3$ field theory [10]), $0.52 \pm 7$ (spin $S,[15]), 0.54 \pm 5$ (DG and $\mathrm{Kl}[17])$ and $0.50 \pm 1\left\{O\left(\varepsilon^{5}\right)[24]\right\}$.

In summary, there no longer seem to be any grounds for doubting that the three-dimensional spin- $\frac{1}{2}$ Ising model obeys hyperscaling and, indeed, belongs to the same universality class as the continuous spin double-Gaussian, Klauder, and $\lambda \phi^{4}$ models [19] which are analytically linked to it : on the contrary, our series analysis provides positive support for the validity of hyperscaling !

\section{Analysis by partial differential approximants.}

In considering the double-Gaussian and Klauder models (or any other similar family) the first question to ask is how many distinct universality classes of critical behaviour are embodied and what is the character of the crossover between different classes as the auxiliary parameter, $y$ in the present case, varies. Single-variable methods of series analysis are intrinsically unsuitable for answering this question in unbiased fashion since, in any crossover regime such methods necessarily predict effective exponents, $\bar{\gamma}(y)$, etc. which vary smoothly with $y$ instead of changing discontinuoysly as unstable multicritical values, say $y_{0}, y_{1}, \ldots$, are crossed.

The PDA technique [26-28] is not subject to this limitation : on the contrary, it can represent properly multicritical behaviour in which the exponents change discretely. Specifically, a function $f(x, y)$ whose power series is known to some order, is approximated by the solution $F(x, y) \equiv[\mathbf{J} / \mathbf{L} ; \mathbf{M}, \mathbf{N} \mid \mathbf{K}]_{f}$, satisfying appropriate boundary conditions, of the defining equation

$$
\begin{aligned}
U_{\mathbf{J}}(x, y)+P_{\mathbf{L}}(x, y) F & = \\
& =Q_{\mathbf{M}}(x, y) \frac{\partial F}{\partial x}+R_{\mathbf{N}}(x, y) \frac{\partial F}{\partial y} .
\end{aligned}
$$


The polynomials $U_{\mathbf{J}}(x, y)$, etc., whose terms $u_{j, j^{\prime}}$ $x^{j} y^{j^{\prime}}, \ldots$ vanish for $\left(j, j^{\prime}\right)$ outside the specified label sets $\mathbf{J}$, etc., are found by solving the generating equation : this is the same as (20) but with $F$ replaced by $f(x, y)$ and error terms of the form $x^{k} y^{k^{\prime}}$ allowed for $\left(k, k^{\prime}\right)$ outside an appropriately chosen matching set $\mathbf{K}[27,28]$. Then a common zero, $\left(x_{0}, y_{0}\right)$, of $Q_{\mathbf{M}}$ and $R_{\mathrm{N}}$ locates a multisingularity of the approximant. Each distinct multisingular point describes a different type of criticality (or multicriticality) specified, in the first place, by exponents $\psi$ and $\theta$ which enter the asymptotic scaling form

$$
f(x, y) \approx|\Delta \tilde{x}|^{-\psi} Z_{f}\left(\Delta \tilde{y} /|\Delta \tilde{x}|^{\phi}\right)+B_{f}(x, y) .
$$

Here the multisingular deviations are given by

$$
\begin{aligned}
& \Delta \tilde{x}=\left(x-x_{0}\right)-\left(y-y_{0}\right) / e_{2} \simeq-K_{0} t, \\
& \Delta \tilde{y}=\left(y-y_{0}\right)-e_{1}\left(x-x_{0}\right),
\end{aligned}
$$

in which $e_{1}$ and $e_{2}$ specify the slopes of the scaling axes, while $B_{f}(x, y)$ represents a smooth background and $Z_{f}(z)$ is the scaling function. The exponents $\psi$ and $\phi$, the slopes $e_{1}$ and $e_{2}$, and the background value $B_{f}\left(x_{0}, y_{0}\right)$ follow from the values of $U, P$ and the derivatives of $Q$ and $R$ at $\left(x_{0}, y_{0}\right)$. When the crossover exponent, $\phi$, is positive the multisingular point is unstable and $\Delta \tilde{y}$ represents a relevant perturbation : this is the situation for the DG and K1 models at the Gaussian multicritical point, $\left(x_{0}, y_{0}\right)=\left(x_{\mathrm{G}}, 0\right)$, for which one knows $\phi=\frac{1}{2}(4-d)$ (for $d \leqslant 4$ ) and $x_{\mathbf{G}}^{\mathrm{bcc}}=\frac{1}{8}$. Conversely when $\phi$ is negative the multisingular point is stable and $\Delta y$ corresponds to an irrelevant variable : then one may put $\theta=-\phi>0$ and expand the scaling function for small $z$ to obtain

$$
\begin{aligned}
& f(x, y) \approx \\
& \quad \approx Z_{0}^{f}|\Delta \tilde{x}|^{-\psi}\left\{1+w_{1}^{f} \Delta \tilde{y}|\Delta \tilde{x}|^{\theta}+\cdots\right\}+B_{0}^{f}+\cdots,
\end{aligned}
$$

where $Z_{0}^{f}=Z(0)$ and $w_{1}^{f}=(d \ln Z / \mathrm{d} z)_{z=0}$. Comparison with (8) shows that $\theta$ has been properly identified as the correction-to-scaling exponent. Furthermore, the correction amplitude is given by

$$
c_{\theta}^{f}(y) \approx w_{1}^{f}\left[1-\left(e_{1} / e_{2}\right)\right]\left(y-y_{0}\right),
$$

and should thus vanish linearly with $y$ when the multisingular point is approached. Note that for a given physical multicritical point the exponent $\phi$ and the slopes $e_{1}$ and $e_{2}$ should be independent of the particular function $f$ studied while $\psi, Z_{f}$ and $B_{f}$ will depend on $f$.

It follows from the solution of the PDA defining equation (20) by integration along trajectories, that the same nonanalytic correction terms with nonvanishing amplitudes, $c_{\theta}^{f}(y)$, must apply along the whole open segment of critical locus, $x_{c}(y)$, which passes through the stable multisingular point in question, say $\left(x_{0}, y_{0}\right) \equiv\left(x_{c}, y_{c}\right)$, and which, in general, is bounded by a pair of adjacent unstable multisingular points. (Reference to Fig. 1 of [17] and Fig. 2 of [19] should prove helpful.)

The strategy for using PDAs to elucidate universality classes is thus clear. In particular, if one constructs PDAs for the susceptibility, $\chi(x, y)$, the sequence and character, stable or unstable, of their multisingular points in the vicinity of the physically anticipated critical locus (or loci) provide an indication of the various types of critical and multicritical behaviour. This program has been implemented for the DG and K1 models [17]. About 300 and 180, respectively, PDAs were computed using coefficients of order $x^{18}$ to $x^{21}$ (and corresponding orders in $y$ ) for different choices of the label sets $\mathbf{J}, \mathbf{L}, \mathbf{M}, \mathbf{N}$ and $\mathbf{K}$. Details are given in [17] and [19] : the approximants selected are invariant or close-to-invariant under Euler transformations on $x$ [28]. In addition, they embody exact information regarding the Gaussian fixed point, which serves as an anchor ensuring accurate behaviour for small $y$.

The first crucial result of the analysis is that the overwhelming majority of the approximants for both models exhibit one and only one stable multisingular point, with coordinate $y_{\mathrm{c}}$, in the range $0<y<1.8$. The values of the exponent $\gamma$ (in the range 1.230 to 1.246) 'and the fact that the critical behaviour of the pure $(y=1)$ Ising - model are controlled by this point enable us to identify it as Ising-like [17]. There is no evidence at all to suggest any intermediate type of critical behaviour, lying between Gaussian and pure Ising, as has been proposed for $d=2$ [29] (but see $[19,30])$ : the whole critical locus, $x_{\mathrm{c}}(y)$, for $0<y \leqslant 1$ is found to be of Ising character.

The observed distribution of the $\gamma$ estimates is quite closely correlated with the distribution of estimates of $y_{c}$ (and of $\theta$ ). For the double-Gaussian model this is illustrated in figure 2 which shows a histogram of $y_{\mathrm{c}}$ estimates and the corresponding ranges of $\gamma$ and $\theta$ estimates. The same plot for the Klauder model, which is found to display appreciably less overall dispersion, appears in [17] together with $\gamma$ and $\theta$ histograms for both models. The comparatively large spread in $y_{\mathrm{c}}$ estimates is directly associated with the stability $(\phi<0)$ and weakly singular character $(\theta \simeq 0.5)$ of the multisingular point ; nevertheless, each estimate, $\left(x_{c}, y_{c}\right)$, lies on, and determines a critical locus, $x_{c}(y)$, which displays very little scatter. By contrast, for $\phi>0$, one finds estimates for the multicritical point which are precise in both coordinates $[26,27]$. To reduce the effects of the dispersion, the PDA data were analysed by forming the mean, $y_{c}$, of the three central quartiles of the histogram and then examining more closely the mean-centred quartile (shown crosshatched in the lower part of figure 2 and shaded in the upper parts) [17]. On this basis the multicritical estimates

$$
y_{\mathrm{c}}^{\mathrm{DG}}=0.87 \pm 4, \quad y_{\mathrm{c}}^{\mathrm{K} \mathbf{l}}=0.81 \pm 6
$$




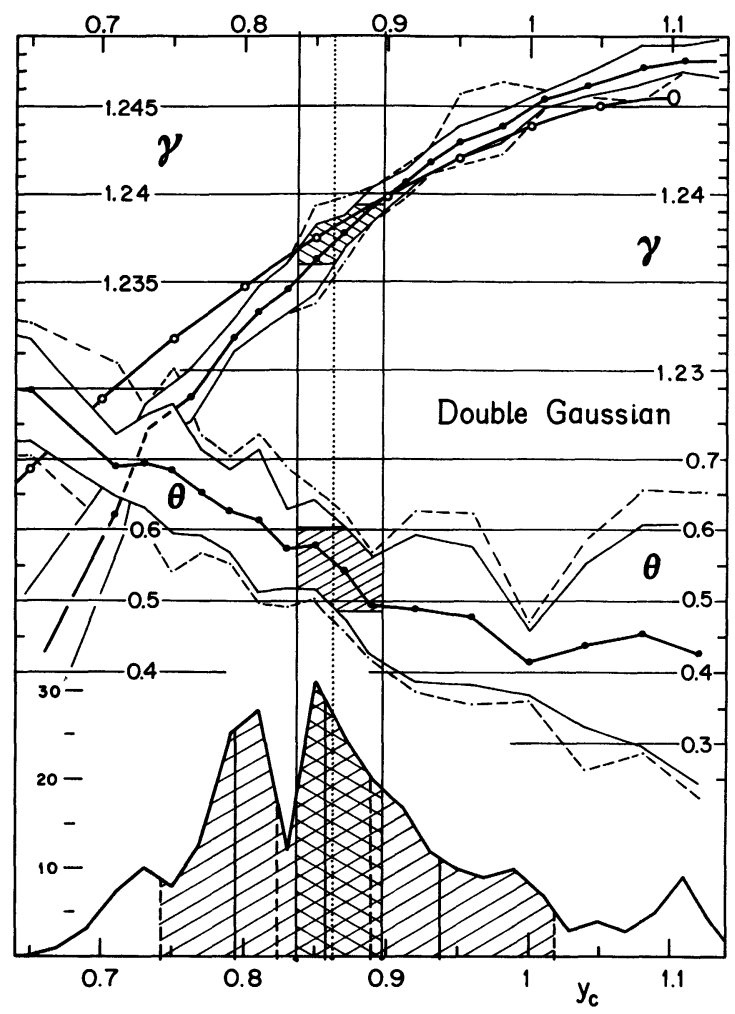

Fig. 2. - Partial differential approximant analysis of the double-Gaussian model showing the histogram of multicritical point estimates, $y_{c}$, and corresponding mean estimates for the exponents $\gamma$ and $\theta$ together with standard deviation limits (see [17]). The open chain plot (circles and dashes) represents the results of a single-variable analysis not allowing for confluent corrections.

were obtained [17]. (The double-Gaussian estimate of George and Rehr [23] is very similar). The corresponding exponent estimates are $\gamma=1.2377 \pm 16(\mathrm{DG})$, $1.2384 \pm 15(\mathrm{Kl})$ and $\theta=0.54 \pm 5(\mathrm{DG})$ and $0.546 \pm 39$ (K1) [17].

The Klauder exponent estimates are probably to be preferred because of the overall lower $y_{\mathrm{c}}$ dispersion. A revised DG central estimate $y_{c}^{\mathrm{DG}}=0.895$, within the range (26), would yield the same $\gamma$ estimate as found for the $\mathrm{K} 1$ model. It is then remarkable, in the light of the cross-model relation (15), that $\left(y_{\mathrm{c}}^{\mathrm{DG}}\right)^{2} \simeq 0.80$ is very close to the central estimate $y_{\mathrm{c}}^{\mathrm{KI}}=0.81$. Thus one sees that the multicritical points in the two different models describe single-spin weight functions with essentially the same fourth moments (the zeroth and second moments, being identical by construction). The actual numerical similarity of the two weight functions for the Klauder range in (26) and corresponding $y_{\mathrm{c}}^{\mathrm{DG}}=0.90$, can be seen in figure 1 . Since the corresponding critical values of $x$ for the two models agree closely (to within 3 or 4 parts in $10^{4}$ ), the effective spin weight functions at criticality (see [19]) are also closely similar. Evidently, we have unearthed a type of quasiuniversality of three-dimensional scalar spin models which supports the notion of a universal
Ising-like fixed point in the full space of scalar spin Hamiltonians. (It should be noted, however, that Hamiltonians equivalent to the fixed point under redundant transformations must be eliminated by appropriate normalizations of the cutoff, the range of quadratic coupling, etc.; it is not entirely clear to us what potential elements of Hamiltonian universality then remain. The issue would seem to be related to the universal block spin distributions discussed by Bruce [31] and Binder [32]).

Finally, note that since we have $y_{\mathrm{c}}<1$ for both models, the behaviour of the pure spin- $\frac{1}{2}$ Ising model at $y=1$ necessarily entails nonvanishing nonanalytic corrections associated with the exponent $\theta$. The trend in estimates visible in figure 2 (see also [17] and below) shows that these corrections tend to increase slightly, by 2 to 4 parts in the third decimal place, the effective exponent $\gamma$ observable in the pure Ising model. The apparent absence of these corrections in the analysis of Ferer and Velgakis [16] seems to be the reason for their somewhat high estimate of $\gamma$. (Calculations are under way to quantify these corrections in more detail at the Ising limit using PDAs).

In order to estimate the exponent $v$ one must study the series for $\mu_{2}(x, y)$ and $\xi^{2}(x, y)$ in the ferromagnetic regime. These series can be investigated by PDA methods and one obtains comparable results for the multicritical location and for the exponents $2 v$ and $\theta$ although the dispersion is somewhat greater than found with $\chi(x, y)$. Likewise the antiferromagnetic locus can be studied using PDAs for all three series in order to estimate $\alpha$. Reasonable results are again found but, owing to the dispersion of estimates (and the complexities of crossover at the antiferromagnetic Gaussian multicritical point) the precision is too low to provide a sharp test of hyperscaling. Accordingly, we will utilise single-variable, fixed-y series to study the issue but allow for their systematic bias in the light of the multisingularity structure revealed by the two-variable analysis.

\section{Critical point estimates}

For the single variable analysis we adopt inhomogeneous differential approximants [21]. For fixed $y$ the approximant $F(x ; y) \equiv[J / L, M]_{f}$ to $f(x, y)$ is the solution of the ordinary differential equation

$$
U_{J}(x)+P_{L}(x) F=Q_{M}(x) \frac{\mathrm{d} F}{\mathrm{~d} x},
$$

satisfying $F(0 ; y)=f(0, y)$. The polynomials $U_{J}$, $P_{L}$ and $Q_{M}$ of degrees $J, L$ and $M$ are calculated, as usual, from the known series for $f(x, y)$ by matching coefficients to as high order as possible. If one sets $U \equiv 0$ (or $J=\varnothing$ ) one simply has D log Padé approximants [21]. The singular points are located by the zeros of $Q(x)$. As mentioned, inhomogeneous approximants can accurately represent functions behaving like (18), i.e. consisting of a single power times an 
analytic amplitude plus a smooth background. However, further confluent nonanalyticities cannot be accurately represented and, if present, will result in systematic deviations in the estimates of critical points and exponents from the true values. Our aim is to capitalize on the systematic character of these deviations which, since the amplitudes of the nonanalytic and analytic corrections vary from one physical property to another, will depend on the particular function analysed as well as on any constraints imposed on the approximants.

An important example of a constraint to be imposed on some of the approximants is

$$
Q(x)=Q(-x)
$$

which implies that $Q$ is actually a polynomial in $x^{2}$ rather than in $x$. This ensures that if $x_{c}(>0)$ is a singularity of an approximant then $x=-x_{\mathrm{c}}$ will also be a singular point. The bipartite character of the bcc lattice implies that the antiferromagnetic and ferromagnetic singularities of the DG and $\mathrm{K} 1$ models are symmetrically related in this way. The latter is a strong singularity in $\chi, \mu_{2}$ and $\xi^{2}$ and so will be more reliably estimated than the former, which is weak. By imposing (28) one thus expects improved accuracy near the antiferromagnetic singularity and, perhaps, even at the ferromagnetic singularity. If one is confident of the location, $x_{c}(y)$ of the ferromagnetic critical point, one can merely impose the constraint

$$
Q\left[-x_{\mathrm{c}}(y)\right]=0,
$$

to a similar end.

We have used the following classes of approximants to estimate the critical locus $x_{\mathrm{c}}(y)$ for the DG and K1 models :

(0) using $\chi(x, y)$ with $U \neq 0$ (adopted as a standard)

(i) using $\mu_{2}(x, y)$ with $U \neq 0$

(ii) using $\chi(x, y)$ with $U \equiv 0$ (D $\log$ Padé)

(iii) using $\chi(x, y)$ with $Q(x)=Q(-x)$

(iv) using $\xi^{2}(x, y)$ with $U \equiv 0$

(v) using $\xi^{2}(x, y)$ with $Q(x)=Q(-x)$.

The individual series were evaluated accurate to 28 decimal places and analysed using quadruple precision. Some of the results for the Klauder model, based in each case on a dozen or so of the highest order approximants, are displayed in table I. (Provided $U_{J} \neq 0$ the estimates are not sensitive to the degree $J$.) For a given model, fixed value of $y$, and particular class of approximants, the precision of the estimates, as gauged from the consistency between different approximants, varies between 2 and 5 parts in $10^{6}$. On the other hand, in the range shown $(y=0.70$ to 0.90 for the Klauder model) systematic differences up to 6 or 7 parts in $10^{5}$ are found between different classes of approximant. The relative deviations from the estimates $x_{\mathrm{c}}^{0}(y)$, namely,

$$
\Delta x_{\mathrm{c}}^{\lambda} / x_{\mathrm{c}}(y) \equiv\left[x_{\mathrm{c}}^{\lambda}(y)-x_{\mathrm{c}}^{0}(y)\right] / x_{\mathrm{c}}^{0}(y),
$$

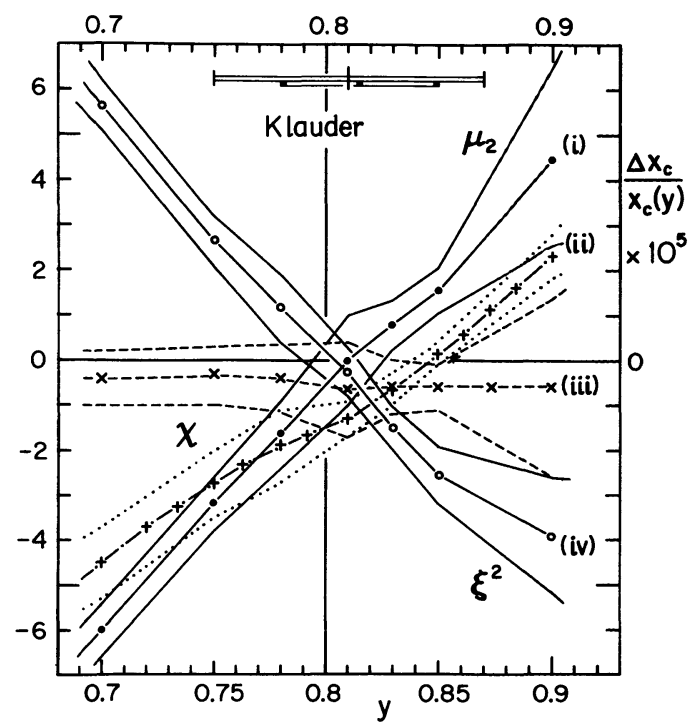

Fig. 3. - Estimates for the critical locus, $x_{\mathrm{c}}(y)$, of the Klauder model based on various classes of differential approximant (see text of Sec. 3) and expressed as differences from the standard estimates, $x_{\mathrm{c}}^{\mathbf{0}}(y)$, derived from unbiased inhomogeneous differential approximants to the susceptibility, $\chi(x, y)$, at fixed $y$. Note that the sign of the differences (iv) has been switched for clarity in the plot.

are plotted in figure 3 together with their apparent uncertainty limits (except that, for clarity, the data for $(\lambda)=(v)$ are not plotted while those for $(\lambda)=(i v)$ are reversed in sign).

One observes a remarkable confluence of all estimates to within one part in $10^{5}$ or less when $y$ is close to $y_{c}^{\mathbf{K}_{1}}=0.815$. Precisely similar behaviour is found for the DG model in the region $y=0.80$ to 1.0. Conservatively, we estimate the confluence regions as

$$
y_{\mathrm{c}}^{\mathrm{DG}}=0.90 \pm 3, \quad y_{\mathrm{c}}^{\mathrm{K} 1}=0.815 \pm 35 .
$$

Evidently these values correspond closely to the multicritical points located in the two-variable PDA analysis see (26) and the horizontal bars in figure 3 . We interpret the agreement between the different estimates when $y^{\mathbf{K} \mathbf{1}} \simeq 0.815$ and $y^{\mathbf{D G}} \simeq 0.90$ as strong evidence that the amplitudes, $c_{\theta}^{f}(y)$ of the leading nonanalytic corrections in $\chi, \mu_{2}$ and $\xi^{2}$ all vanish in the close neighbourhood, in agreement with the expected scaling forms and the conclusion (25). Estimates of exponents for these values of $y$ should thus be close to the true values. Note also, in relation to the discussion at the end of the previous section, that the values (32) correspond closely under the transformation (15).

The central values in (32) lead to the multicritical point estimates

$$
8 x_{\mathrm{c}}^{\mathrm{DG}}=1.29073 \pm 1, \quad 8 x_{\mathrm{c}}^{\mathrm{Kl}}=1.21062 \pm 1 .
$$

If the uncertainties in (32) are allowed for, the uncertainties quoted here must be increased by 1 or 2 parts 
in $10^{5}$ and a systematic increase in $x_{\mathrm{c}}$ with $y_{\mathrm{c}}$, corresponding to the critical locus slopes

$$
8\left(\mathrm{~d} x_{\mathrm{c}} / \mathrm{d} y\right) \equiv 8 / e_{2} \simeq 0.465(\mathrm{DG}), \quad 0.260(\mathrm{Kl}),
$$

must also be recognized. However, the values of the multicritical locations have no universal significance so we turn directly to the exponent estimates.

\section{Exponents and hyperscaling.}

The estimates for the exponents $v$ and $\alpha$ obtained from various classes of approximant and for different values of $y$ are summarized graphically in figures 4 and 5 for the double-Gaussian and Klauder models, respectively. Further and more detailed results are

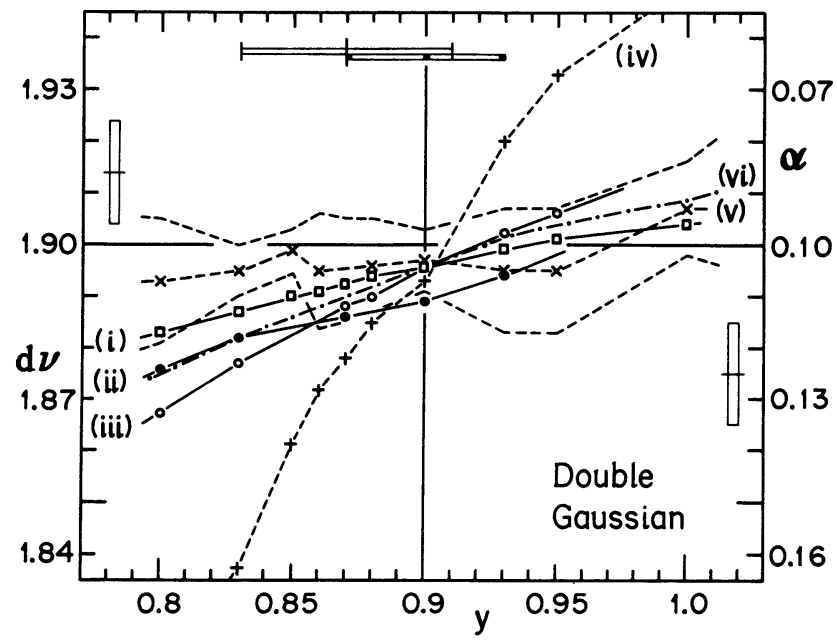

Fig. 4. - Estimates for the exponent $v$, (i), (ii) and (iii), and for the exponent $\alpha$, (iv), (v) and (vi), for the double-Gaussian model as obtained from various classes of differential approximants : see text of section 4 . Note that the vertical scales are aligned so that $d v=2-\alpha$. The open boxes at the two sides indicate the old $v$ and $\alpha$ estimates which suggested the violation of hyperscaling.

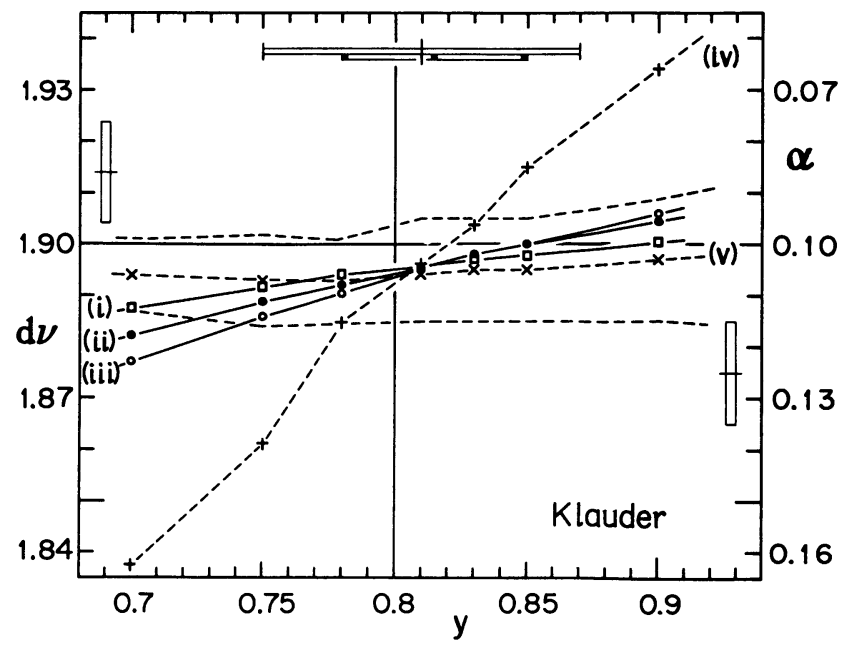

Fig. 5. - Estimates of the exponents $v$ and $\alpha$ for the Klauder model, as in figure 4 , indicating the validity of hyperscaling.

in table I where some of the Klauder model data are presented numerically. The table lists, in particular, the standard estimates, $\gamma^{0}(y)$, for the susceptibility : these display a smoothly increasing trend with $y$. (Compare also with the open chain curves, circles and links, in figure 2 and in figure 3 of [17]). The corresponding $\gamma$ estimates for the approximants of classes (ii) and (iii) (of the previous section) behave similarly but increase at a slightly different rate with $y$, a confluence then being observed in the vicinity of $y_{\mathbf{c}}^{\mathbf{K l}}$; likewise for the DG model. The central values in (32) then yield $\gamma \simeq 1.2395$ with last-place uncertainties of \pm 3 and \pm 4 for the DG and Kl models, respectively, so justifying the overall estimate quoted in (19). Changes in the value adopted for $y_{c}$ within the range specified in (32) lead to correlated changes of \pm 12 in the last place of the $\gamma$ estimate. However, we believe that this full uncertainty range is overconservative.

Table I. - Single-variable estimates for the Klauder model of critical points, $x_{\mathrm{c}}(y)$, and effective exponents as a function of $y$. As in figure 3 the superscripts indicate : (0) "standard" using $\chi$ series and general inhomogeneous approximants; (i) using $\mu_{2}$ and general approximants; (iv) using $\xi^{2}$ and homogeneous approximants $(U \equiv 0)$; in addition $2 v^{\mathrm{i} / 0} \equiv(\gamma+2 v)^{\mathrm{i}}-\gamma^{0}$. The apparent uncertainties in the $x_{\mathrm{c}}$ estimates vary from 2 to 6 in the last decimal place shown but for the exponents are only 1 or 2 in the last place. (For clarity the first four digits of $x_{\mathrm{c}}^{\mathrm{i}}$ and $x_{\mathrm{c}}^{\text {iv }}$ have been omitted.)

\begin{tabular}{c|r|r|r|r|r|r|r}
\hline$y$ & 0.70 & \multicolumn{1}{|c|}{0.75} & 0.78 & 0.81 & 0.83 & 0.85 & \multicolumn{1}{c}{0.90} \\
\hline $8 x_{\mathrm{c}}^{\mathrm{o}}(y)$ & 1.180790 & 1.193736 & 1.201523 & 1.209326 & 1.214532 & 1.219746 & 1.232805 \\
$8 x_{\mathrm{c}}^{\mathrm{i}}(y)$ & 719 & 698 & 503 & 326 & 542 & 765 & 860 \\
$8 x_{\mathrm{c}}^{\mathrm{iv}}(y)$ & 723 & 704 & 509 & 329 & 550 & 777 & 858 \\
\hline$\gamma^{0}(y)$ & 1.2366 & 1.2380 & 1.2389 & 1.2396 & 1.2402 & 1.2406 & 1.2416 \\
$(\gamma+2 v)^{\mathrm{i}}$ & 2.4879 & 2.4953 & 2.4993 & 2.5032 & 2.5053 & 2.5074 & 2.5124 \\
$2 v^{\mathrm{i} / 0}$ & 1.2513 & 1.2573 & 1.2604 & 1.2636 & 1.2651 & 1.2668 & 1.2708 \\
$2 v^{\mathrm{iv}}$ & 1.2549 & 1.2592 & 1.2616 & 1.2638 & 1.2652 & 1.2665 & 1.2702 \\
\hline
\end{tabular}


Table I also lists the estimates of the compound exponent $(\gamma+2 v)$ obtained from $\mu_{2}$ and, by combining this with the estimates $\gamma^{0}(y)$, the corresponding estimates for $2 v$ (labelled $2 v^{\mathrm{i} / 0}$ ). These latter values may be compared directly with the estimates, labelled $2 v^{\text {iv }}$, obtained from the $\xi^{2}$ series using homogeneous (i.e. $\mathrm{D} \log$ ) approximants. It is seen that the two routes to estimating $v$ yield very similar results in the vicinity of the multicritical point $y_{\mathbf{c}}^{\mathbf{K}}$. Again quite comparable behaviour is found for the double-Gaussian model.

Turning, finally, to figures 4 and 5 note, first, that the lefthand and righthand vertical scales are aligned so that $d v(\equiv 3 v)=2-\alpha$, in order to test hyperscaling: the open boxes on the left and right sides of the figures represent the original pure Ising model estimates (5), which suggested that hyperscaling was violated. At the top of each figure the upper open bar indicates the PDA estimate (26) for the multicritical point, $y_{c}$; the lower bar represents the present conclusion (32), based on the confluence of estimates for $x_{c}(y)$ (see Fig. 3). Each figure displays three independent estimates for $d v$ derived as follows :

(i) from $\xi^{2}(x, y)$ with $Q(x)=Q(-x)$ imposed

(ii) from $\xi^{2}(x, y)$ with $U \equiv 0$ (i.e. $\mathrm{D} \log$ Padé)

(iii) from $\mu_{2}(x, y)$ using $\gamma^{0}(y)$ [labelled (iv) in table I].

The precision of the estimates for $d v$ is as good as 2 or 3 parts in the fourth decimal place for (i) and (ii), which is too small to indicate on the graphs ! Owing partly to the differencing entailed, the uncertainties in the estimates of class (iii) are two to three times larger. As can be seen from the figures, the three sets of estimates increase smoothly with $y$ but agree to within \pm 0.001 or better, when $y^{\mathrm{DG}}=0.90$ and $y^{\mathrm{Kl}}=0.815$. Indeed, it is striking that the confluence of these estimates is even sharper, in terms of $y$, than that of the $x_{\mathrm{c}}(y)$ estimates ! From the DG data we thus estimate $d v=1.895 \pm 3$; the $\mathrm{Kl}$ data indicate $d v=1.896 \pm 4$. The close agreement is gratifying and justifies the $v$ estimate quoted in (19). Again, however, a change in the assigned value of $y_{c}$ results in correlated changes in the $d v$ estimates amounting, for the range (32), to about \pm 5 and \pm 4 in the third place for DG and $\mathrm{Kl}$ models, respectively.

Lastly, figures 4 and 5 display estimates of $\alpha$ determined by the antiferromagnetic singularity in approximants derived from :

(iv) $\xi^{2}(x, y)$ with $Q(x)=Q(-x)$;

(v) $\chi(x, y)$ with $Q(x)=Q(-x)$;

(vi) $\mu_{2}(x, y)$ with $Q\left(-\left|x_{\mathrm{c}}\right|\right)=0$ imposed.
The estimates (v), for which the uncertainty range is also displayed, show comparatively little variation with $y$. By contrast the estimates (iv), derived from $\xi^{2}$, vary surprisingly rapidly for both models (and are less precise by factors of 2 to 3 ). Nevertheless, the intersection of the estimates (iv) and (vi) once more occurs close to the central multicritical estimates ! The data for $y=y_{\mathrm{c}}$ indicate $\alpha=0.104 \pm 7$ for the DG model (or a slightly greater uncertainty if one allows for changes in the $y_{\mathrm{c}}$ estimate) and $\alpha=0.105 \pm 10$ for the $\mathrm{Kl}$ model ; on this basis we reach the conclusion quoted in (19). For reasons that are not well understood, the behaviour of $\mu_{2}$-based estimates for $\alpha$ are not as well behaved. As an illustration, the plot (vi) is presented for the double-Gaussian model : the antiferromagnetic critical point was imposed here using the standard ferromagnetic estimates (based on $\chi$ ). The estimates for $\alpha$ at $y \simeq 0.90$ are slightly higher than (iv) and (v) but are still encompassed by the conclusion (19). It would, however, clearly be of interest to have high order series for the specific heat itself in the hope of gaining more precise estimates for $\alpha$.

To conclude, note that the estimates (19) imply $\omega^{*} v \equiv d v-(2-\alpha)=0.001 \pm 10$ : thus hyperscaling is confirmed to well within the uncertainties. Indeed, an $a$ priori believer in hyperscaling might be inclined to think that our quoted uncertainty limits are too conservative ! However, even if the leading nonanalytic correction vanishes close to our estimates of $y_{\mathrm{c}}$, the remaining higher order nonanalyticities have been accounted for only in some average way so that we should not be overconfident as to the accuracy of the final exponent estimates despite the precision of the check on hyperscaling.

\section{Acknowledgments.}

We are indebted to Professor B. G. Nickel for giving us the series expansions on which our work is based. Our researches have been supported by grants from the National Science Foundation through the Applied Mathematics Program with ancillary support through the Materials Science Center at Cornell University. One of us (M.E.F.) is grateful to Professor R. J. Elliott and the Department of Theoretical Physics at Oxford University for hospitality and to the Science and Engineering Research Council of the United Kingdom for partial support while this paper was being written.
[1] Fisher, M. E., Rep. Prog. Phys. 30 (1967) 615.

[2] Fisher, M. E., Proc. Nobel Symp. 24, Collective Properties of Physical Systems, edited by B. Lundqvist and S. Lundqvist (New York, Academic Press) 1974, p. 16.
[3] Domв, C. Phase Transitions and Critical Phenomena 3, edited by C. Domb and M. S. Green (New York, Academic Press) 1974.

[4] Baker, G. A., Jr., Phys. Rev. B 15 (1977) 1552. 
[5] For a review see : Fisher, M. E., Rev. Mod. Phys. 46 (1974) 597.

[6] Fisher, M. E. Advanced Course on Critical Phenomena Lecture Notes in Physics 186, edited by F. J. W. Hahne (Berlin, Springer-Verlag) 1983 : see especially Appendix D.

[7] Brézin, E., Le Guillou, J. C. and Zinn-Justin, J., Phase Transitions and Critical Phenomena edited by C. Domb and M. S. Green (New York, Academic Press) 1976, vol. 6.

[8] FISHER, M. E., Renormalization Group and Quantum Field Theory edited by J. D. Gunton and M. S. Green (Philadelphia, Temple University) 1974, p. 65 .

[9] BaKer, G. A., Jr., Nickel, B. G., Green, M. S. and MeIron, D. I., Phys. Rev. Lett. 36 (1976) 1351 ;

BAKer, G. A., Jr., Nickel, B. G. and MeIron, D. I., Phys. Rev. B 17 (1978) 1365.

[10] Le Guillou, J. C. and Zinn-Justin, J., Phys. Rev. Lett. 39 (1977) 95 ; Phys. Rev. B 21 (1980) 3976.

[11] Zinn-Justin, J., J. Physique 40 (1979) 969.

[12] Nickel, B. G. and Sharpe, B., J. Phys. A 12 (1979) 1819.

[13] ReHr, J. J., J. Phys. A 12 (1979) L179.

[14] Nickel, B. G. Phase Transitions-Cargese 1980, edited by M. Levy, J. C. Le Guillou and J. Zinn-Justin (New York, Plenum Publ. Corp.) 1982, p. 291.

[15] Zinn-Justin, J., J. Physique 42 (1981) 783.

[16] Ferer, M. and VelgaKis, M. J., Phys. Rev. B 27 (1983) 2839.

[17] Chen, J.-H., Nickel, B. G. and Fisher, M. E., Phys. Rev. Lett. 48 (1982) 630. Note that the expression for $b^{2}(y)$ is misprinted in this reference : see Eq. (14) here.

[18] Barma, M., J. Phys. A 16 (1983) L745 and private communication.
[19] Barma, M. and Fisher, M. E., Phys. Rev. B 31 (1985) 5954.

[20] Baker, G. A., Jr. and Bishop, A. R., J. Phys. A 15 (1982) L201.

[21] Fisher, M. E. and Au-YANG, H., J. Phys. A 12 (1979) 1677 ; ibid. 13 (1980) 1517;

Hunter, D. L. and Baker, G. A., Jr., Phys. Rev. B 19 (1979) 3808.

[22] Fisher, M. E., Philos. Mag. 7 (1962) 1731 ;

LANGer, J. S. and Fisher, M. E., Phys. Rev. Lett. 20 (1968) 665.

[23] George, M. J. and Rehr, J. J., Phys. Rev. Lett. 53 (1984) 2063.

[24] Vladimirov, A. A., Kazakov, D. I. and Tarasov, O. V., Sov. Phys. JETP 50 (1979) 521;

Goroshny, S. G., Lavin, S. A. and Tkachov, F. V., Phys. Lett. A 101 (1981) 120.

[25] Pawley, G. S., Swendsen, R. H., Wallace, D. J. and Wilson, K. G., Phys. Rev. B 29 (1984) 4030.

[26] Fisher, M. E. and KerR, R. M., Phys. Rev. Lett. 39 (1977) 667.

[27] Fisher, M. E. and Chen, J.-H. Phase TransitionsCargese 1980, edited by M. Levy, J. C. Le Guillou, and J. Zinn-Justin (New York, Plenum Publ. Corp.) 1982.

[28] Styer, D. F. and Fisher, M. E., Proc. R. Soc. A 384 (1982) 259 ; 388 (1983) 75.

[29] Baker, G. A., Jr. and Johnson, J. D., J. Phys. A 17 (1984) L275 ; Phys. Rev. Lett. 54 (1985) 2461.

[30] Barma, M. and Fisher, M. E., Phys. Rev. Lett. 53 (1984) 1935 ; ibid. 54 (1985) 2462.

[31] Bruce, A. D., J. Phys. C 14 (1981) 3667.

[32] Binder, K., Phys. Rev. Lett. 47 (1981) 693 ; Z. Phys. B43 (1981) 119. 